\title{
ON THE TENSOR SQUARE OF NON-ABELIAN NILPOTENT FINITE DIMENSIONAL LIE ALGEBRAS
}

\author{
PEYMAN NIROOMAND
}

\begin{abstract}
For every finite $p$-group $G$ of order $p^{n}$ with derived subgroup of order $p^{m}$, Rocco in [10] proved that the order of tensor square of $G$ is at most $p^{n(n-m)}$. This upper bound has been improved recently by author in [9]. The aim of the present paper is to obtain a similar result for a non-abelian nilpotent Lie algebra of finite dimension. More precisely, for any given $n$-dimensional non-abelian nilpotent Lie algebra $L$ with derived subalgebra of dimension $m$ we have $\operatorname{dim}(L \otimes L) \leq(n-m)(n-1)+2$. Furthermore for $m=1$, the explicit structure of $L$ is given when the equality holds.
\end{abstract}

\section{INTRODUCTION}

The theory of tensor product of groups has been developed for Lie algebras by Ellis in [4, 6. A question naturally can be asked here: which results on tensor square of groups, holds for Lie algebras? every partial answer to this question may be an interesting result in the theory of Lie algebras. Several authors have been obtained in [1, 2, 4, 5, 6, 17, 8, 12, similar argument to known result in group theory. In this article we focus on a result of Rocco [10 which states for every finite $p$-group of order $p^{n}$ with derived subgroup of order $p^{m}$, the order of its tensor square is at most $p^{n(n-m)}$. Salemkar et. al. [12] proved the same statement for Lie algebras of finite dimension. Recently the author [9], improved the upper bound of Rocco and proved the order of tensor square is at most $p^{(n-1)(n-m)+2}$. Furthermore when $m=1$ the structure of groups under which the equality holds is completely described. The aim of this paper is to show the similar inequality does hold for nilpotent Lie algebras of finite dimension. As easily seen the ratio of two mentioned upper bounds is $p^{n-(m+2)}$ which is a considerable quantity so the result of this paper sharpens the inequality in [12] a considerable amount.

\section{TENSOR PRODUCT AND SOME KNOWN RESULTS}

In this section first we introduce notation and terminology which are used in this paper. Then we summarize some known results without proof which will be used throughout the paper.

Throughout this paper let $F$ be a fixed field and [,] denotes the Lie bracket. For any two arbitrary Lie Algebras $M$ and $N$, an action of $M$ on $N$ means a $F$-bilinear map $M \times N \longrightarrow N$ sending $(m, n)$ to ${ }^{m} n$ satisfying

$$
{ }^{\left[m, m^{\prime}\right]} n={ }^{m}\left({ }^{m^{\prime}} n\right)-{ }^{m^{\prime}}\left({ }^{m} n\right) \text { and }{ }^{m}\left[n, n^{\prime}\right]=\left[{ }^{m} n, n^{\prime}\right]+\left[n,{ }^{m} n^{\prime}\right],
$$

for all $m, m^{\prime} \in M$ and $n, n^{\prime} \in N$.

Key words and phrases. nilpotent Lie algebra; tensor square; Schur multiplier.

Mathematics Subject Classification 2010. Primary 17B30; Secondary 17 B60. 
Lie multiplication in a Lie algebra $M$ can induce an action on itself via ${ }^{m^{\prime}} m=$ $\left[m^{\prime}, m\right]$ for all $m, m^{\prime} \in M$. Also for any Lie algebra $L$ a bilinear function $h$ : $M \times N \longrightarrow L$ is called a Lie pairing if for all $m, m^{\prime} \in M$ and $n, n^{\prime} \in N$ provided that

$$
\begin{aligned}
& h\left(\left[m, m^{\prime}\right], n\right)=h\left(m,{ }^{m^{\prime}} n\right)-h\left(m^{\prime},{ }^{m} n\right), \\
& h\left(m,\left[n, n^{\prime}\right]\right)=h\left({ }^{n^{\prime}} m, n\right)-h\left({ }^{n} m, n^{\prime}\right), \\
& h\left({ }^{n} m,{ }^{m^{\prime}} n^{\prime}\right)=-\left[h(m, n), h\left(m^{\prime}, n^{\prime}\right)\right] .
\end{aligned}
$$

The tensor product $M \otimes N$ is the Lie algebra generated by the symbols $m \otimes n(m \in$ $M, n \in N)$ subject to the following relations:

(i) $c(m \otimes n)=c m \otimes n=m \otimes c n$,

(ii) $\left(m+m^{\prime}\right) \otimes n=m \otimes n+m^{\prime} \otimes n, m \otimes\left(n+n^{\prime}\right)=m \otimes n+m \otimes n^{\prime}$,

(iii) $\left[m, m^{\prime}\right] \otimes n=m \otimes\left({ }^{m^{\prime}} n\right)-m^{\prime} \otimes\left({ }^{m} n\right), m \otimes\left[n, n^{\prime}\right]=\left({ }^{n^{\prime}} m\right) \otimes n-\left({ }^{n} m\right) \otimes n^{\prime}$,

(iv) $\left[(m \otimes n),\left(m^{\prime} \otimes n^{\prime}\right)\right]=-\left(n^{m}\right) \otimes\left({ }^{m^{\prime}} n^{\prime}\right)$ for all $c \in F, m, m^{\prime} \in M$ and $n, n^{\prime} \in N$.

Let $M$ and $N$ be two Lie algebras acting on each other. We are able to find more results on the non-abelian tensor product $M \otimes N$ by putting a condition on these actions. The actions are called compatible if

$$
\left.{ }^{(n} m\right) n^{\prime}=\left[n^{\prime},{ }^{m} n\right] \text { and }{ }^{\left({ }^{m} n\right)} m^{\prime}=\left[m^{\prime},{ }^{n} m\right] \text { for all } m, m^{\prime} \in M, n, n^{\prime} \in N .
$$

In the case, $M=N$ and all actions are given by Lie multiplication, then $M \otimes M$ is called tensor square of $M$. The exterior product $L \wedge L$ is obtained from $L \otimes L$ by imposing the additional relation $l \otimes l$ for all $l$ in $L$ and the image of $l \otimes l^{\prime}$ is denoted by $l \wedge l^{\prime}$ for all $l, l^{\prime} \in L$ (see [5, 4]).

By terminology of [6], let $L \square L$ be the submodule of $L \otimes L$ generated by the elements $l \otimes l$. One can check that $L \square L$ lies in the centre of $L \otimes L$ and $L \wedge L \cong$ $L \otimes L / L \square L$.

Ellis in [5] showed that, $\mathcal{M}(L)$, the Schur multiplier of $L$, is isomorphic to the Kernel $\left(L \wedge L \longrightarrow L^{2}, l \wedge l^{\prime} \longrightarrow\left[l, l^{\prime}\right]\right)$. He also in [6] generalized the universal quadratic functor $\Gamma$ (see $[13$ for more details). The quadratic functor $\Gamma$ let us study the relation between the Lie exterior product and the Lie tensor product.

Now we mention the following consequence of propositions from [6, 13, 12, for the convenience of the reader.

Proposition 2.1. (See [6, Proposition 15, 16]). If $M$ and $N$ act trivially on each other, then there is an isomorphism

$$
M \otimes N \cong M \bigotimes_{\bmod } N, \text { where } M \bigotimes_{\bmod } N
$$

is the standard tensor product of $M$ and $N$.

Proposition 2.2. (See [6, Proposition 15, 16] and [13]).

(i) For any two Lie algebras $M$ and $N$ there is an isomorphism

$$
\Gamma(M \oplus N)=\Gamma(M) \oplus \Gamma(N) \oplus M \bigotimes_{\bmod } N .
$$

(ii) There is an isomorphism $\Gamma(F) \cong F$.

Proposition 2.3. (See [12, Remark 1.5 (d)] and [6. Proposition 14, 15]). Let L be a Lie algebra, Then : 
ON THE TENSOR SQUARE OF NON-ABELIAN NILPOTENT FINITE DIMENSIONAL LIE ALGEBRA\&

(i) If $N \subseteq L^{2} \cap Z(G)$, then the sequence

$$
N \otimes L \longrightarrow L \otimes L \longrightarrow L / N \otimes L / N \longrightarrow 0
$$

is exact.

(ii) The sequence

$$
\Gamma\left(L / L^{2}\right) \stackrel{\psi}{\longrightarrow} L \otimes L \stackrel{\pi}{\longrightarrow} L \wedge L \longrightarrow 0
$$

is exact.

(iii) In particular, if $L / L^{2}$ is a free Lie algebra, then the sequence

$$
0 \longrightarrow \Gamma\left(L / L^{2}\right) \stackrel{\psi}{\longrightarrow} L \otimes L \stackrel{\pi}{\longrightarrow} L \wedge L \longrightarrow 0
$$

is exact.

The following proposition provides the behavior of tensor product with direct sums.

Proposition 2.4. (See [6, Proposition 8]). There is an isomorphism

$(M \oplus N) \otimes(M \oplus N) \cong(M \otimes M) \oplus(M \otimes N) \oplus(N \otimes M) \oplus(N \otimes N)$.

Recall that a finite dimensional Lie algebra $L$ is called Heisenberg if $L^{2}=Z(L)$ and $\operatorname{dim} L^{2}=1$. Such algebras are odd dimensional with basis $v_{1}, \ldots, v_{2 m}, v$ and the only non-zero multiplication between basis elements is $\left[v_{2 i-1}, v_{2 i}\right]=-\left[v_{2 i}, v_{2 i-1}\right]=$ $v$ for $i=1, \ldots m$. The symbol $H(m)$ denotes the Heisenberg algebra of dimension $2 m+1$. The tensor square of Heisenberg algebra will be characterized in the next section by using the following result.

Lemma 2.5. (See [2, Example 3] and [8, Theorem 24]).

(i) $\operatorname{dim}(\mathcal{M}(H(1)))=2$.

(ii) $\operatorname{dim}(\mathcal{M}(H(m)))=2 m^{2}-m-1$ for all $m \geq 2$.

Let $t(L)=\frac{1}{2} n(n-1)-\operatorname{dim} \mathcal{M}(L)$ and $A(n)$ denoted abelian Lie algebra of dimension $n$. In [1, 7] the structure on $L$ has been characterized only by the size of $\mathcal{M}(L)$ as follows.

Theorem 2.6. (See [1, Lemmas 2, 3 and Theorems 3, 5] and [7, Theorem 1]). Let $L$ be an $n$-dimensional nilpotent Lie algebra. Then

(i) $t(L) \geq 0$;

(ii) $t(L)=0$ if and only if $L \cong A(n)$;

(iii) $t(L)=1$ if and only if $L \cong H(1)$;

(iv) $t(L)=2$ if and only if $L \cong H(1) \oplus A(1)$;

(v) $t(L)=3$ if and only if $L \cong H(1) \oplus A(2)$.

\section{Main Results}

In this section at first we obtain the tensor square of Heisenberg algebras. Then we show that any $n$-dimensional non-abelian nilpotent Lie algebra $L$ with derived subalgebra of dimension $m$ satisfies

$$
\operatorname{dim}(L \otimes L) \leq(n-m)(n-1)+2 .
$$

In addition for $m=1$, we obtain the explicit structure of $L$ when the equality holds.

Lemma 3.1. For any two abelian Lie algebras $M$ and $N$ there is an isomorphism

$$
(M \oplus N) \square(M \oplus N) \cong M \square M \oplus N \square N \oplus M \otimes N .
$$


Proof. Since Proposition 11 given in [3] carries over to the case of Lie algebra (see Proposition 2.4). Hence the similar proof of group theory given in [11, Remark 5] carries over to the case of Lie algebra.

Corollary 3.2. Let $L$ be a finite dimension Lie algebra, then

$$
\Gamma\left(L / L^{2}\right) \cong L \square L \cong L / L^{2} \square L / L^{2} .
$$

Proof. By invoking Proposition $2.3(i i), \operatorname{Im} \psi=L \square L$. On the other hand, Proposition 2.3 (iii) implies that $\Gamma(A(1)) \cong A(1) \square A(1) \cong A(1)$. The rest of proof is obtained by the fact that there is a natural epimorphism $L \square L \longrightarrow L / L^{2} \square L / L^{2}$, Proposition 2.2 (i) and Lemma 3.1.

Proposition 3.3. Let $H(m)$ be a Heisenberg Lie algebra, then

$$
H(m) \otimes H(m) \cong H(m) / H(m)^{2} \otimes H(m) / H(m)^{2},
$$

when $m \geq 2$. In the case $m=1, H(1) \otimes H(1)$ is an abelian Lie algebra of dimension 6.

Proof. First suppose that $m \geq 2$. According to the Lemmas 2.5, 3.1 and Corollary 3.2 ,

$$
\begin{aligned}
\operatorname{dim}(H(m) \otimes H(m)) & =\operatorname{dim}(H(m) \square H(m))+\operatorname{dim} \mathcal{M}(H(m))+\operatorname{dim} H(m)^{2} \\
& =\frac{1}{2} 2 m(2 m+1)+2 m^{2}-m-1+1 \\
& =4 m^{2} .
\end{aligned}
$$

On the other hand, $\operatorname{dim}\left(H(m) / H(m)^{2} \otimes H(m) / H(m)^{2}\right)=4 m^{2}$. Hence the result obtained by the natural Lie epimorphism $H(m) \otimes H(m) \longrightarrow H(m) / H(m)^{2} \otimes$ $H(m) / H(m)^{2}$.

In the case $m=1$, by a similar fashion $H(1) \otimes H(1)$ is a Lie algebra of dimension 6 . One can easily check that $H(1) \otimes H(1)$ is abelian, which completes the proof.

Theorem 3.4. Let $L$ be an $n$-dimensional non-abelian nilpotent Lie algebra with derived subalgebra of dimension $m$. Then

$$
\operatorname{dim}(L \otimes L) \leq(n-m)(n-1)+2 .
$$

In particular, for $m=1$ the equality holds if and only if $L \cong H(1) \oplus A(n-3)$.

Proof. First suppose that $m=1$, since $L^{2} \subseteq Z(L)$ by using Proposition 3.3, we may assume that $L^{2} \subsetneq Z(L)$. Thus $L^{2}$ has a complement $A$ in $Z(L)$. The same argument shows that $Z(L) / L^{2}$ has a complement $H / L^{2}$ in $L / L^{2}$. One can check that $H$ is a Heisenberg Lie algebra and $L=H \oplus A$. Hence by Proposition 2.4,

$$
\operatorname{dim}((H \oplus A) \otimes(H \oplus A))=\operatorname{dim}(H \otimes H)+2 \operatorname{dim}(H \otimes A)+\operatorname{dim}(A \otimes A)
$$

On the other hands, Proposition 2.1 implies that

$$
H \otimes A \cong H / H^{2} \bigotimes_{\bmod } A
$$

Two cases may be considered respect to the $\operatorname{dim} H$.

Case $I$. If $H \cong H(1)$, then by invoking of Propositions 2.4 and 3.3, we have

$$
\begin{aligned}
\operatorname{dim}((H \oplus A) \otimes(H \oplus A)) & =6+4(n-3)+(n-3)^{2} \\
& =(n-1)^{2}+2 .
\end{aligned}
$$


Case $I I$. If $H \cong H(m)$, then

$$
\begin{aligned}
\operatorname{dim}((H \oplus A) \otimes(H \oplus A)) & =4 m^{2}+4 m(n-2 m-1)+(n-2 m-1)^{2} \\
& =(n-1)^{2} .
\end{aligned}
$$

Let $m \geq 2$ and consider an ideal $N$ contained in $Z(L) \cap L^{2}$, induction hypothesis and Proposition 2.3 ( $i$ ) deduce that

$$
\operatorname{dim}(L \otimes L) \leq \operatorname{dim}(L / N \otimes L / N)+\operatorname{dim}(N \otimes L) .
$$

Now by virtue of Proposition 2.1 .

$$
\operatorname{dim}(N \otimes L)=\operatorname{dim}\left(N \bigotimes_{\bmod } L / L^{2}\right)
$$

Hence

$$
\operatorname{dim}(L \otimes L) \leq n-m+(n-m)(n-2)+2=(n-1)(n-m)+2,
$$

as required.

Proposition 3.5. Let $L$ be an $n$-dimensional non-abelian nilpotent Lie algebra with derived subalgebra of dimension $m(n \geq 4, m \geq 2)$. Then

$\operatorname{dim}(L \otimes L) \leq(n-m)(n-1)+1$ or $\operatorname{dim}(L \otimes L) \leq(n-m)(n-1)+2<n(n-m)$.

Proof. Let $\operatorname{dim}(L \otimes L)>(n-m)(n-1)+1$, thus by Theorem 3.4, we should have $\operatorname{dim}(L \otimes L)=(n-m)(n-1)+2$. By contrary we assume that $\operatorname{dim}(L \otimes L)=n(n-m)$. Hence $m+n=2$.

First suppose that $n=4$. Since $m=2$, Theorem 2.6 implies that $\operatorname{dim} \mathcal{M}(L) \leq 2$ and hence $\operatorname{dim}(L \otimes L) \leq 7$. Now let $n \geq 5$, induction hypothesis and Proposition $2.3(i)$ implies that

$$
\operatorname{dim}(L \otimes L) \leq(n-m)(n-1)+1,
$$

which is a contradiction hence the result obtained.

The last proposition shows developed Rocco's bound for a nilpotent Lie algebra is less than the bound in Theorem 2.6 except for the case $L \cong H(1)$.

\section{REFERENCES}

[1] P. Batten, K. Moneyhun, and E. Stitzinger, On characterizing nilpotent Lie algebras by their multipliers, Comm. Algebra. (24)14 (1996), 4319-4330.

[2] P. Batten and E. Stitzinger, On covers of Lie algebras, Comm. Algebra (24)14 (1996), 43014317.

[3] R. Brown, D.L. Johnson, and E.F. Robertson, Some Computations of non Abelian tensor products of groups, J. Algebra 111 (1987), 177-202.

[4] G. Ellis, Crossed modules and their higher dimensional analogues, Ph.D. thesis, University of Wales, 1984.

[5] G. Ellis, Nonabelian exterior products of Lie algebras and an exact sequence in the homology of Lie algebras. J. Pure Appl. Algebra 46 (1987), 111-115.

[6] G. Ellis, A nonabelian tensor product of Lie algebras, Glasgow Math. J. 33 (1991), 101-120.

[7] P. Hardy and E. Stitzinge, On characterizing nilpotent Lie algebras by their multipliers $t(L)=$ 3, 4, 5, 6, Comm. Algebra (26)11 (1998), 3527-3539.

[8] K. Moneyhun, Isoclinisms in Lie algebras, Algebras Groups Geom. 11 (1994), 9-22.

[9] P. Niroomand, Non abelian tensor square of non abelian prime power groups, submitted.

[10] N.R. Rocco, On a construction related to the nonabelian tensor square of a group, Bol. Soc. Brasil. Mat. (22)1 (1991) 63-79. 
[11] N. R. Rocco, A presentation for a crossed embedding of finite solvable groups, Comm. Algebra (22)6 (1994), 1975-1998.

[12] A.R. Salemkar, H. Tavallaee, H. Mohammadzadeh, and B. Edalatzadeh, On the non-abelian tensor product of Lie algebras, Linear Multilinear Algebra 1 (2009), 1-9.

[13] J.H.C. Whitehead, A certain exact sequence, Ann. of Math. 52 (1950), 51-110.

School of Mathematics and Computer Science, Damghan University of Basic Sciences, Damghan, Iran

E-mail address: niroomand@dubs.ac.ir 